\title{
Hadronic structure from double parton scattering
}

\author{
Matteo Rinaldi ${ }^{1, *}$ and Federico Alberto Ceccopieri ${ }^{2}$ \\ ${ }^{1}$ Departamento de Física Teoórica-IFIC, Universidad de Valencia-CSIC, \\ 46100 Burjassot (Valencia), Spain \\ ${ }^{2}$ IFPA, Université de Liège, B4000, Liège, Belgium
}

(Received 22 January 2018; published 27 April 2018)

\begin{abstract}
In the present paper we consider the so-called effective cross section, a quantity which encodes the experimental knowledge on double parton scattering in hadronic collisions that has been accumulated so far. We show that the effective cross section, under some assumptions close to those adopted in its experimental extractions, can be used to obtain a range of mean transverse distance between an interacting parton pair in double parton scattering. Therefore, we have proved that the effective cross section offers a way to access information on the hadronic structure.
\end{abstract}

DOI: 10.1103/PhysRevD.97.071501

\section{INTRODUCTION}

A proper description of the event structure in hadronic collisions requires the inclusion of so-called multiple parton interactions (MPI), which affect both the multiplicity and topology of the hadronic final state [1,2]. The large hadron collider operation renewed the interest in MPI given the continuous demand for an increasingly detailed description of the hadronic final state, which is crucial in many new physics searches. In this rapidly evolving context, these types of studies have received attention for their own sake: they might be sensitive to partonic correlations in the colliding hadrons. The simplest MPI process is double parton scattering (DPS). In such a case, a large momentum transfer is involved in both scatterings and perturbative techniques can be applied to calculate the corresponding cross section. The latter depends on a two-body nonperturbative quantity, the so-called double parton distribution functions (dPDFs). These distributions are interpreted as number densities of parton pairs with a given transverse distance, $b_{\perp}$, in coordinate space and carrying longitudinal momentum fractions $\left(x_{1}, x_{2}\right)$ of the parent proton [3]. Double PDFs are not calculable from first principles, a feature shared with ordinary PDFs and other nonperturbative quantities in QCD. However, due to their dependence upon the partonic interdistance [4], they contain information on the hadronic structure complementary to those obtained from one-body distributions such as generalized parton distribution functions (GPDs) and transverse

\section{*mrinaldi@ific.uv.es}

Published by the American Physical Society under the terms of the Creative Commons Attribution 4.0 International license. Further distribution of this work must maintain attribution to the author(s) and the published article's title, journal citation, and DOI. Funded by SCOAP. momentum dependent PDFs. Unfortunately, since the DPS cross section depends on an integral over $b_{\perp}$, there are no experimental observables which may give direct access to such a dependence [1].

In this scenario, calculations of dPDFs via hadronic models have been used to obtain basic information and to gauge the impact of longitudinal and transverse correlations [5-9]. Despite this wealth of information possibly encoded in dPDFs, the experimental knowledge on DPS cross section has been accumulated, up to now, into the socalled effective cross section, $\sigma_{\text {eff }}$. The latter is defined through the ratio of the product of two single parton scattering cross sections to the DPS cross section with the same final states. The effective cross section has been extracted, although in a model dependent way, in several experiments; see recent results in Refs. [10-15]. The purpose of the present paper is to demonstrate that, by exploiting the maximum information encoded in $\sigma_{\text {eff }}$ and by using almost the same assumptions used in its experimental extraction, a range of mean distances, characterizing the interacting parton pair, can be derived. Thanks to this result, one can access information on the hadronic structure, encoded in the dPDFs $b_{\perp}$ dependence, in a quite rather easy way without any detailed knowledge on such a dependence in transverse space. Therefore, the present analysis represents an attempt to generalize historical studies on the proton radius in exclusive processes to the relative partonic distance between two interacting partons in DPS processes in hadronic collisions.

This paper is organized as follows. In Sec. II, we show how, in principle, novel information on the proton structure can be achieved by means of dPDFs and a new "form factor" is introduced. In Sec. III, we elaborate a general relation between $\sigma_{\text {eff }}$ and the mean distance of two interacting partons. In Secs. III A and III B, we derive a couple of inequalities suitable to extract information on the 
mean partonic distance from experimental values of $\sigma_{\text {eff }}$. In Sec. IV, we discuss numerical results. We collect our conclusions in Sec. V.

\section{HADRON STRUCTURE VIA DPS}

Similarly to the case of GPDs, whose first moment is related to standard Dirac and Pauli form factors, we may introduce the first moment of dPDFs with respect to $x_{1}$ and $x_{2}$,

$$
f_{i j}\left(k_{\perp}\right)=\frac{1}{N_{i j}} \int d x_{1} d x_{2} F_{i j}\left(x_{1}, x_{2}, k_{\perp}\right),
$$

where $i$ and $j$ are parton indices and we address $f_{i j}\left(k_{\perp}\right)$ as the "effective form factor" [16]. The functions $F_{i j}\left(x_{1}, x_{2}, k_{\perp}\right)$ are the Fourier transform of dPDFs $\tilde{F}_{i j}\left(x_{1}, x_{2}, b_{\perp}\right)$. According to Ref. [17], the $N_{i j}$ factors in Eq. (1) represent the dPDF normalizations evaluated at $k_{\perp}=0$, e.g., for valence quarks $N_{u_{v} d_{v}}=N_{u_{v} u_{v}}=2$. At variance with the GPD case, here $k_{\perp}$ does not represent a momentum transfer between the proton initial and final state but rather a transverse momentum imbalance between two partons in the amplitude and its conjugate [18]. Therefore, in momentum space $F_{i j}\left(x_{1}, x_{2}, k_{\perp}\right)$ does not admit a probabilistic interpretation, which holds instead in $b_{\perp}$ space. The effective form factor can be defined in a more fundamental manner in terms of the proton wave function. In fact, in the nonrelativistic limit, it is given by

$$
\begin{aligned}
f_{i j}\left(k_{\perp}\right)= & \int d \vec{k}_{1} d \vec{k}_{2} \Psi^{\dagger}\left(\vec{k}_{1}+\vec{k}_{\perp}, \vec{k}_{2}\right) \\
& \times \tau_{i} \tau_{j} \Psi\left(\vec{k}_{1}, \vec{k}_{2}+\vec{k}_{\perp}\right)
\end{aligned}
$$

with $\Psi\left(\vec{k}_{1}, \vec{k}_{2}\right)$ being the canonical proton wave function in the intrinsic frame depending on the parton momentum $\vec{k}_{i}$ and $\tau_{i}$ the usual flavor projector, see, e.g., Ref. [6]. The effective form factor can be related to the two-body density of partons, $\tilde{f}_{i j}\left(b_{\perp}\right)$, with $b_{\perp}$ being the relative distance between two partons, defined by means of the Fourier transform of the proton wave function with respect to $\vec{k}_{\perp}$, i.e.,

$$
f_{i j}\left(k_{\perp}\right)=\int d \vec{b}_{\perp} e^{i \vec{k}_{\perp} \cdot \vec{b}_{\perp}} \tilde{f}_{i j}\left(b_{\perp}\right) .
$$

Equations (2) and (3) are similar to those used to define the standard electromagnetic proton form factor in terms of the same hadron wave function $\Psi\left(\vec{k}_{1}, \vec{k}_{2}\right)$. Analogously to this standard case, one can define the mean value of the distance between two partons in the transverse plane through the effective form factor,

$$
\left\langle b^{2}\right\rangle_{i j} \simeq-\left.4 \frac{d f_{i j}\left(k_{\perp}\right)}{d k_{\perp}^{2}}\right|_{k_{\perp}=0}
$$

The knowledge of $f_{i j}\left(k_{\perp}\right)$ gives access to new information, generalizing the results on the proton mean radius, obtained from electromagnetic proton form factors in elastic processes. Despite the richness of information encoded in the effective form factor, this quantity is actually poorly known from the theoretical and experimental points of view. In fact, in DPS processes, only information on the integral of dPDFs with respect to $k_{\perp}$ is available [1]. In order to overcome this problem, in the next sections we present a procedure which relates the mean partonic distance between two partons directly to the experimentally extracted $\sigma_{\text {eff }}$.

\section{III. $\sigma_{\text {eff }}$ AND PARTONIC DISTANCES}

The differential DPS cross section, assuming that the two hard scattering processes can be factorized [3,18-21], involves dPDFs through an integral over $k_{\perp}$ and reads [18]

$$
\begin{aligned}
d \sigma_{\mathrm{DPS}}^{A+B}= & \frac{m}{2} \int \frac{d^{2} k_{\perp}}{(2 \pi)^{2}} d \hat{\sigma}_{i k}^{A} d \hat{\sigma}_{j l}^{B} . \\
& \cdot F_{i j}\left(x_{1}, x_{2}, k_{\perp}\right) F_{k l}\left(x_{3}, x_{4},-k_{\perp}\right) .
\end{aligned}
$$

It represents the Fourier-transformed version of the DPS cross section formula in $b_{\perp}$ space presented in Ref. [1]. In Eq. (5), $d \hat{\sigma}$ are the differential partonic cross sections for processes $\mathrm{A}$ and $\mathrm{B}$, respectively, and the symmetry factor $m=1$ if $A=B$, and $m=2$ otherwise. Given the limited knowledge regarding dPDFs, a fully factorized ansatz is frequently assumed,

$$
F_{i j}\left(x_{1}, x_{2}, k_{\perp}\right) \sim q_{i}\left(x_{1}\right) q_{j}\left(x_{2}\right) f\left(k_{\perp}\right),
$$

where $q_{i}(x)$ are ordinary PDFs. Usually, in such a simplified approach, the transverse form factor, $f\left(k_{\perp}\right)$, depends neither on parton flavors nor on its fractional momenta [22]. It is worth mentioning that dPDF calculations within hadronic models show, in general, a breaking of the factorized ansatz, Eq. (6), in a specific region of phase space, where sizable longitudinal and mixed longitudinal-transverse partonic correlations do appear [5-9]. Nevertheless, in this paper we still the use the approximation in Eq. (6) in order to make contact with experimental extractions of $\sigma_{\text {eff }}$. We remark, however, that in the present work, no assumptions on the detailed functional form of $f\left(k_{\perp}\right)$ are used. In such a case, the DPS cross section simplifies to the form [4]

$$
d \sigma_{\mathrm{DPS}}^{A+B}=\frac{m}{2} \frac{d \sigma_{\mathrm{SPS}}^{A} d \sigma_{\mathrm{SPS}}^{B}}{\sigma_{\mathrm{eff}}},
$$


with $d \sigma_{\mathrm{SPS}}^{A}(B)$ being the single parton scattering cross section with final state $A(B)$. In this scenario, $\sigma_{\text {eff }}$ is simply given in $k_{\perp}$ space by

$$
\sigma_{\mathrm{eff}}^{-1}=\int \frac{d^{2} k_{\perp}}{(2 \pi)^{2}} f\left(k_{\perp}\right)^{2}=\int \frac{d k_{\perp}}{2 \pi} k_{\perp} f\left(k_{\perp}\right)^{2},
$$

where the last expression follows from rotational invariance since we are interested in scattering processes whose final states are integrated over angles. Equation (7) shows that $\sigma_{\text {eff }}$ enters the DPS cross section formula as an overall normalization factor. Starting from Eq. (8), we show in this section how such an integral can be related to the mean distance of the two partons involved in the scattering process. For this purpose, we use two properties granted from the general structure of the hadronic wave function in Eq. (2), i.e.,

$$
f\left(k_{\perp}=0\right)=1 \quad \text { and } \quad f\left(k_{\perp} \rightarrow \infty\right)=0 .
$$

Thanks to the latter conditions, two identities are immediately obtained,

$$
\int_{0}^{\infty} d k_{\perp} k_{\perp}^{m} f\left(k_{\perp}\right)^{2}=-2 \int_{0}^{\infty} d k_{\perp} \frac{k_{\perp}^{m+1}}{m+1} f\left(k_{\perp}\right) \frac{d}{d k_{\perp}} f\left(k_{\perp}\right),
$$

with $m \geq 0$ and

$$
\int_{0}^{\infty} d k_{\perp} f\left(k_{\perp}\right)^{s-1} \frac{d}{d k_{\perp}} f\left(k_{\perp}\right)=-\frac{f(0)^{s}}{s}=-\frac{1}{s},
$$

which will be frequently used in the following. Furthermore, with $\vec{k}_{\perp}$ being defined on the transverse plane, in two dimensions, $f\left(k_{\perp}\right)$ can be defined as

$$
\begin{aligned}
f\left(k_{\perp}\right) & =\int d^{2} b_{\perp} e^{i \vec{k}_{\perp} \cdot \vec{b}_{\perp}} \tilde{f}\left(b_{\perp}\right) \\
& =2 \pi \int d b \tilde{f}(b) J_{0}\left(k_{\perp} b\right),
\end{aligned}
$$

with $\tilde{f}(b)$ being the probability density of finding two partons with a relative transverse distance $b=\left|\vec{b}_{\perp}\right|, \tilde{f}(b)$ being a radial function of $b$. By expanding in series the Bessel function $J_{0}\left(k_{\perp} b\right)$, we find the following useful representation:

$$
f\left(k_{\perp}\right)=\sum_{n=0}^{\infty} k_{\perp}^{2 n}\left\langle b^{2 n}\right\rangle \frac{(-1)^{n}}{4^{n}(n !)^{2}}=\sum_{n=0}^{\infty} k_{\perp}^{2 n}\left\langle b^{2 n}\right\rangle P_{n}^{J_{0}}
$$

where the $P_{n}^{J_{0}}$ are the coefficients of the Bessel expansion and $\left\langle b^{2 n}\right\rangle$ are the $2 n$ moments of $\tilde{f}(b)$ and contain all dynamical unknown information on partonic proton structure. At this point, we arrange Eq. (8) in a form more suitable for our purposes. We consider Eq. (11) for $s=3$ and, by using the expansion in Eq. (13) with the $n=0$ and $n=1$ terms kept explicit, we get

$$
\begin{aligned}
-\frac{1}{3} & =\int_{0}^{\infty} d k_{\perp} f\left(k_{\perp}\right)^{2} f^{\prime}\left(k_{\perp}\right) \\
& =\int_{0}^{\infty} d k_{\perp} f\left(k_{\perp}\right) f^{\prime}\left(k_{\perp}\right)\left[1-\frac{k_{\perp}^{2}\left\langle b^{2}\right\rangle}{4}+\sum_{n=2} P_{n}^{J_{0}} k_{\perp}^{2 n}\left\langle b^{2 n}\right\rangle\right] .
\end{aligned}
$$

The terms in square brackets are then evaluated as follows. The first one is simplified by using Eq. (11) with $s=2$, the second one by using Eq. (10) with $m=1$, and the last term by using Eq. (10) with $m=2 n-1$. Collecting results and dividing by $\left\langle b^{2}\right\rangle / 4$, we find

$$
\begin{aligned}
& \int_{0}^{\infty} d k_{\perp} k_{\perp} f\left(k_{\perp}\right)^{2} \\
& \quad=\frac{2}{3\left\langle b^{2}\right\rangle}+4 \sum_{n=2} \frac{\left\langle b^{2 n}\right\rangle P_{n}^{J_{0}} n}{\left\langle b^{2}\right\rangle} \int_{0}^{\infty} d k_{\perp} k_{\perp}^{2 n-1} f\left(k_{\perp}\right)^{2} .
\end{aligned}
$$

Although Eq. (15) shows a formal relation between $\sigma_{\text {eff }}$ and $\left\langle b^{2}\right\rangle$, the latter is obscured by the last term, which requires the explicit knowledge of $f\left(k_{\perp}\right)$. In the next two subsections, we show how this problem can actually be circumvented, providing an easy-to-evaluate relation between $\sigma_{\text {eff }}$ and $\left\langle b^{2}\right\rangle$. We mention here for later convenience that by a repeated use of the Cauchy-Schwarz inequality and the property of the variance, $\left\langle b^{2}\right\rangle \geq\langle b\rangle^{2}$, it can be easily shown that

$$
\left\langle b^{n}\right\rangle \geq\langle b\rangle^{n}
$$

which represents a generalization of the property of the variance for $n \geq 2$.

\section{A. A minimum for the allowed partonic distance}

In this subsection, we show how, given a known value for $\sigma_{\text {eff }}$, a minimum value for the mean partonic distance can be derived by using Eq. (15). For this purpose, generalizing Eq. (4), we introduce the function

$$
d_{2}\left(k_{\perp}\right)=-2 f^{\prime}\left(k_{\perp}\right) / k_{\perp} .
$$

By using the expansion for $f\left(k_{\perp}\right)$ in Eq. (13), one finds

$d_{2}\left(k_{\perp}\right)=-4 \sum_{n=1} k_{\perp}^{2 n-2}\left\langle b^{2 n}\right\rangle P_{n}^{J_{0}} n=\left\langle b^{2}\right\rangle+\mathcal{O}\left(k_{\perp}^{2}\right)$,

which immediately gives $d_{2}\left(k_{\perp}=0\right)=\left\langle b^{2}\right\rangle$. At this point, one may notice that the formal definition of $f\left(k_{\perp}\right)$, Eq. (2), is rather similar to the one of the electromagnetic 
proton form factor, except that in the present case $k_{\perp}$ is a transverse momentum imbalance in a two-body distribution. Since electromagnetic proton form factors are, in general, decreasing functions of $k_{\perp}$, we may expect a similar behavior in $f\left(k_{\perp}\right)$. This observation implies that $d_{2}\left(k_{\perp}\right) \geq 0$ via Eq. (17). Additionally, one may notice that

$$
\left.\frac{d}{k_{\perp} d k_{\perp}} d_{2}\left(k_{\perp}\right)\right|_{k_{\perp}=0}=-8 P_{2}^{J_{0}}<0
$$

implying that $d_{2}(0)$ is a maximum for $d_{2}\left(k_{\perp}\right)$. At this point, one may consider the identity in Eq. (11) with $s=3$,

$$
\int_{0}^{\infty} d k_{\perp} k_{\perp} f\left(k_{\perp}\right)^{2} d_{2}\left(k_{\perp}\right)=2 / 3 .
$$

Since $d_{2}(0)$ is a maximum for $d_{2}\left(k_{\perp}\right)$, we deduce from Eq. (20) that

$$
\int_{0}^{\infty} d k_{\perp} k_{\perp} f\left(k_{\perp}\right)^{2} \geq \frac{2}{3\left\langle b^{2}\right\rangle},
$$

a result which can be rewritten in terms of the effective cross section as $\left\langle b^{2}\right\rangle \geq \sigma_{\text {eff }} /(3 \pi)$. We remark that the same result can be obtained starting directly from Eq. (15). In fact, thanks to the variance property in Eq. (16) and the formal definition of $P_{n}^{J_{0}}$, one can analytically prove that the second term on the right-hand side of Eq. (15) is positive, therefore leading to the same final result, Eq. (21).

\section{B. A maximum for the allowed partonic distance}

In this subsection, we investigate whether $\sigma_{\text {eff }}$ determines a maximum value for the mean interpartonic distance. We note that the properties of $f\left(k_{\perp}\right)$ used up to now will not be sufficient for our purpose, and we will introduce additional reasonable assumptions which we will discuss during the proof.

From the definition of $\sigma_{\text {eff }}$ in Eq. (8), we note that the integral is positive definite; thus, we can introduce an integer $\tilde{N}$ such that

$$
\frac{2 \pi}{\sigma_{\text {eff }}}=\int_{0}^{\infty} d k_{\perp} k_{\perp} f\left(k_{\perp}\right)^{2}=\frac{1}{\tilde{N}\left\langle b^{2}\right\rangle} .
$$

Therefore, for any $N \leq \tilde{N}$,

$$
\int_{0}^{\infty} d k_{\perp} k_{\perp} f\left(k_{\perp}\right)^{2} N\left\langle b^{2}\right\rangle \leq 1 .
$$

Trivially, $N=0$ is a solution of this equation, which is of no interest. However, given the result in Eq. (21), our problem reduces to the search of a nonzero value of $N$ in the range $0<N<3 / 2$. For this purpose, we subtract from Eq. (23) the identity in Eq. (11) with $s=2$, obtaining

$$
\int_{0}^{\infty} d k_{\perp} k_{\perp} f\left(k_{\perp}\right)\left[N\left\langle b^{2}\right\rangle f\left(k_{\perp}\right)-d_{2}\left(k_{\perp}\right)\right] \leq 0 .
$$

Finding a solution to Eqs. (23) and (24) is not possible without detailed knowledge of the functional form of $f\left(k_{\perp}\right)$. Nevertheless, we can study the sign of the term in square brackets in Eq. (24), i.e.,

$$
N\left\langle b^{2}\right\rangle f\left(k_{\perp}\right) \leq d_{2}\left(k_{\perp}\right) .
$$

This inequality represents a sufficient condition for the validity of Eq. (24). The condition in not necessary because there might exist regions in $k_{\perp}$ and values of $N$ for which such a term is positive but the integral in Eq. (24) is negative. To further proceed, let us rewrite Eq. (25) by using the series expansion of $f\left(k_{\perp}\right)$ and $d_{2}\left(k_{\perp}\right)$, obtaining

$$
N\left\langle b^{2}\right\rangle \sum_{n=0} P_{n}^{j_{0}} k_{\perp}^{2 n}\left\langle b^{2 n}\right\rangle \leq \sum_{n=0} \frac{P_{n}^{j_{0}}}{n+1} k_{\perp}^{2 n}\left\langle b^{2 n+2}\right\rangle .
$$

By equating terms of equal powers in $k_{\perp}$, we get the following set of solutions:

$$
\frac{1}{n+1}<N<\frac{1}{n}, \quad n=\text { odd }
$$

Such solutions, however, do not take into account the detailed $k_{\perp}$ - dependence of $f\left(k_{\perp}\right)$. For example, if the integral in Eq. (22) is dominated by the low $k_{\perp}$ region, the solution to Eqs. (24) and (25) is found in the first interval, namely $1 / 2<N<1$. Since this case corresponds to an effective form factor falling sufficiently fast at large $k_{\perp}$, we take this condition as a working hypothesis and provide supporting arguments in the following.

In the first place, we wish to quantify the limiting asymptotics of $f\left(k_{\perp}\right)$ at large $k_{\perp}$, which satisfies the proposed solution. For this purpose, we consider a dipole test function of the type

$$
f\left(k_{\perp}\right)=\left(1+\frac{k_{\perp}^{2}}{m^{2}}\right)^{-r}
$$

in which $m$ is a mass parameter and the large $k_{\perp}$ behavior is controlled by the tunable parameter $r$. By direct evaluation, we find that our proposed solution is valid if $r>1$ in Eq. (28). The same result holds for functions that fall even faster at large $k_{\perp}$ like Gaussians and exponentials.

Secondly, additional support for the proposed solution is provided by the following observation [23]: $f\left(k_{\perp}\right)$ represents a two-body form factor, $k_{\perp}$ being a transverse momentum imbalance between the parton pair. As such, its asymptotic behavior at large $k_{\perp}$ should fall more rapidly than the one in one-body form factors. If one uses for $f\left(k_{\perp}\right)$ the results obtained in Refs. [24-26], one finds that the proposed solution is verified since these functions all have 
dipole forms with $r=2$. The same conclusion is reached if Dirac and Pauli form factors are used, whose behaviors at large momentum transfer $Q$ are given by $1 / Q^{4}$ and $1 / Q^{6}$, respectively [27]. Finally, we remark that the proposed solution is found to be valid for model calculations of $f\left(k_{\perp}\right)$, in particular the one evaluated within the LightFront approach in Ref. [7] and for the two-gluon form factor discussed in Ref. [23]. To conclude, we have found that Eq. (24) is verified for $N=1 / 2$ under the additional condition that $f\left(k_{\perp}\right)$ falls off as $k_{\perp}^{-2}$ or faster. As a consequence of our derivation we can state that

$$
\int_{0}^{\infty} d k_{\perp} k_{\perp} f\left(k_{\perp}\right)^{2} \leq \frac{2}{\left\langle b^{2}\right\rangle} .
$$

Combining this result with Eq. (21) leads to

$$
\frac{\sigma_{\text {eff }}}{3 \pi} \leq\left\langle b^{2}\right\rangle \leq \frac{\sigma_{\text {eff }}}{\pi},
$$

which limits the range of the interpartonic distance and is the main result of the paper. We wish to close this section by highlighting the degree of model dependence of this result. The latter indeed does depend on the approximations made in Eqs. (5) and (6), in particular on the full factorization of $f\left(k_{\perp}\right)$ in the dPDF expression together with its flavor and energy dependence. Therefore, it contains the same model dependence assumed in the $\sigma_{\text {eff }}$ extraction. However, our result does depend weakly on the details of $f\left(k_{\perp}\right)$, since just the general conditions in Eq. (9) and its limiting asymptotics at large $k_{\perp}$ are assumed, leaving the detailed shape $f\left(k_{\perp}\right)$ largely unconstrained.

\section{NUMERICAL RESULTS}

In this section, we discuss a direct application of Eq. (30). Since the latter is derived with a set of assumptions close to the ones used by experimental collaborations to extract $\sigma_{\text {eff }}$, we are allowed to use a representative selection of DPS processes with different final states and rather different kinematics. In particular, we consider the DPS production of double quarkonia and of high mass final states, since this final state discrimination appears to be correlated with the extracted value of $\sigma_{\text {eff }}$. Therefore, we consider recent LHC analyses in which $\sigma_{\text {eff }}$ is extracted in the double $J / \Psi$ channel by the LHCb [10], ATLAS [14], and by the authors of Ref. [15] based on CMS data, in the 4-jets channel by ATLAS [11], and in the $W+2$ jets and same sign $W W$ channels analyzed by CMS $[12,13]$. Results are presented in Fig. 1, where the range of allowed mean partonic distance has been calculated according to Eq. (30) and displayed with inner bars. The theoretical uncertainty $\Delta$ associated with Eq. (30), defined as the difference between the upper and lower limit of $\left\langle b^{2}\right\rangle$, parametrizes the ignorance of the details of $f\left(k_{\perp}\right)$. The latter does depend linearly on $\sigma_{\text {eff }}$ so $\Delta$ gets smaller as $\sigma_{\text {eff }}$ decreases, a

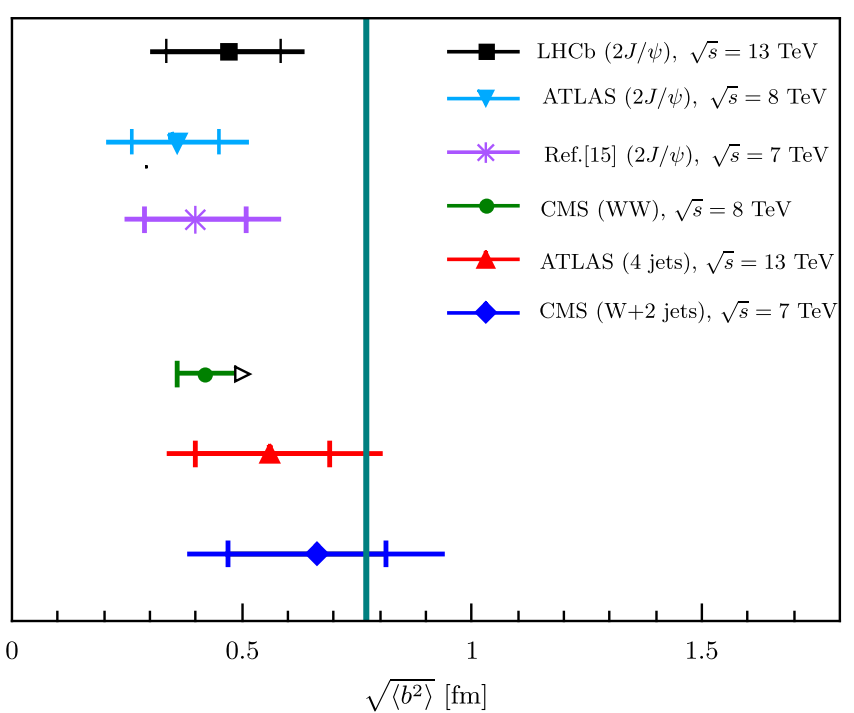

FIG. 1. The range of allowed mean partonic distance, Eq. (30), calculated by using $\sigma_{\text {eff }}$ extracted values from different experimental analyses [10-15]. Inner error bars represent the theoretical uncertainty associated with the range in Eq. (30). The outer ones represent the propagation of experimental uncertainties, related to $\sigma_{\text {eff }}$ extraction, plus theoretical ones added in quadrature. The vertical line represents the proton radius.

trend which can be observed in Fig. 1. Taking into account the experimental uncertainties associated with the $\sigma_{\text {eff }}$ extraction and adding them in quadrature to the theoretical ones, we obtain the outer error bars. We conclude that, by using the extracted values of $\sigma_{\text {eff }}$ and their corresponding errors, our estimate of the allowed range of $\left\langle b^{2}\right\rangle$ via Eq. (30) is dominated by the theoretical uncertainty, a conclusion that comes as no surprise since our result is obtained without assuming any detailed knowledge of the shape of $f\left(k_{\perp}\right)$. It is worth noticing that the upper limit on the partonic distance for $\sigma_{\text {eff }}<20 \mathrm{mb}$ is substantially smaller than the electromagnetic radius of the proton. This is a posteriori confirmation that measured values of $\sigma_{\text {eff }}$ are not compatible with trivial expectations based on geometrical considerations, and they directly point to dynamical correlation effects in the proton; see the discussion in [28].

In particular, we have found a minimum for the distance in the range $0.2<\sqrt{\left\langle b^{2}\right\rangle_{\min }}<0.35 \mathrm{fm}$, which is driven by $\sigma_{\text {eff }}$ extracted from processes involving heavy quarkonia pairs in the final state. On the other hand, the maximum varies in the range $0.6<\sqrt{\left\langle b^{2}\right\rangle_{\max }}<0.95 \mathrm{fm}$ and is driven by $\sigma_{\text {eff }}$ extracted from processes involving electroweak bosons and/or jets. We point out that our mathematical approach works even if $\sigma_{\text {eff }}$ is not constant among different processes since it is sufficient that Eq. (8) holds. Therefore, DPS measurements with final states whose production is dominated by distinct flavor species will, hopefully, allow the investigation of the flavor dependence of $\sigma_{\text {eff }}$ and consequently of the effective form factor. 


\section{CONCLUSIONS}

In the present paper we have presented a method which allows us to convert the information encoded in $\sigma_{\text {eff }}$, a derived quantity often used in experimental analyses to characterize the DPS cross section, into information on the partonic proton structure. The procedure used here makes a number of assumptions which are close to the ones frequently used in experimental analyses. Furthermore, we make no use of the detailed knowledge of the transverse form factor and only assume its reasonable behavior at very large $k_{\perp}$, guided by studies on the standard proton form factors. In this way, the experimentally extracted $\sigma_{\text {eff }}$ can be directly cast into a range of mean distances characterizing the interacting parton pair.

\section{ACKNOWLEDGMENTS}

This work was supported in part by Mineco under Contracts No. FPA2013-47443-C2-1-P, Mineco and UE Feder under Contracts No. FPA2016-77177-C2-1-P, GVA-PROMETEOII/2014/066 and SEV-2014-0398. We warmly thank Sergio Scopetta, Marco Traini, and Vicente Vento for many useful discussions and comments on the manuscript.
[1] N. Paver and D. Treleani, Nuovo Cimento Soc. Ital. Fis. 70A, 215 (1982).

[2] T. Sjostrand and M. van Zijl, Phys. Lett. B 188, 149 (1987); Phys. Rev. D 36, 2019 (1987).

[3] M. Diehl and A. Schafer, Phys. Lett. B 698, 389 (2011); M. Diehl, D. Ostermeier, and A. Schafer, J. High Energy Phys. 03 (2012) 089.

[4] G. Calucci and D. Treleani, Phys. Rev. D 60, 054023 (1999).

[5] H. M. Chang, A. V. Manohar, and W. J. Waalewijn, Phys. Rev. D 87, 034009 (2013).

[6] M. Rinaldi, S. Scopetta, and V. Vento, Phys. Rev. D 87, 114021 (2013).

[7] M. Rinaldi, S. Scopetta, M. Traini, and V. Vento, J. High Energy Phys. 12 (2014) 028.

[8] M. Rinaldi, S. Scopetta, M. Traini, and V. Vento, J. High Energy Phys. 10 (2016) 063.

[9] M. Rinaldi and F. A. Ceccopieri, Phys. Rev. D 95, 034040 (2017).

[10] R. Aaij et al. (LHCb Collaboration), J. High Energy Phys. 06 (2017) 047; 20 (2017) 068(E).

[11] M. Aaboud et al. (ATLAS Collaboration), J. High Energy Phys. 11 (2016) 110.

[12] S. Chatrchyan et al. (CMS Collaboration), J. High Energy Phys. 03 (2014) 032.

[13] A. M. Sirunyan et al. (CMS Collaboration), J. High Energy Phys. 02 (2018) 032.
[14] M. Aaboud et al. (ATLAS Collaboration), Eur. Phys. J. C 77, 76 (2017).

[15] J. P. Lansberg and H. S. Shao, Phys. Lett. B 751, 479 (2015).

[16] M. Rinaldi, S. Scopetta, M. Traini, and V. Vento, Phys. Lett. B 752, 40 (2016).

[17] J. R. Gaunt and W. J. Stirling, J. High Energy Phys. 03 (2010) 005.

[18] B. Blok, Y. Dokshitzer, L. Frankfurt, and M. Strikman, Phys. Rev. D 83 (2011) 071501; Eur. Phys. J. C 72, 1963 (2012); 74, 2926 (2014).

[19] M. Diehl, J. R. Gaunt, D. Ostermeier, P. Ploessl, and A. Schafer, J. High Energy Phys. 01 (2016) 076.

[20] M. Diehl, J. R. Gaunt, and K. Schonwald, J. High Energy Phys. 06 (2017) 083.

[21] M. G. A. Buffing, M. Diehl, and T. Kasemets, J. High Energy Phys. 01 (2018) 044.

[22] M. Mekhfi, Phys. Rev. D 32 (1985) 2371.

[23] L. Frankfurt and M. Strikman, Phys. Rev. D 66, 031502 (2002).

[24] J. C. Bernauer et al. (A1 Collaboration), Phys. Rev. Lett. 105, 242001 (2010).

[25] L. Andivahis et al., Phys. Rev. D 50, 5491 (1994).

[26] O. Gayou et al. (Jefferson Lab Hall A Collaboration), Phys. Rev. Lett. 88, 092301 (2002).

[27] G. P. Lepage and S. J. Brodsky, Phys. Rev. D 22, 2157 (1980).

[28] D. Treleani, Phys. Rev. D 76, 076006 (2007). 